\title{
ELISA KIT EVALUATION FOR IGG AND IGM ANTIBODIES TO A-60 TUBERCULAR PROTEIN ANTIGEN
}

Y. KALANTRI, N. HEMVANI, G. C. BHATIA, D. S. CHITNIS

\section{ABSTRACT}

AIMS: The purpose of this study is to evaluate the A-60 antigen-based enzyme-linked immuno sorbent assay (ELISA) test for its sensitivity, specificity, and other related statistical parameters. SETTINGS AND DESIGN: Sera from 114 healthy volunteers, 105 bacteriologically confirmed cases of pulmonary tuberculosis (PTB), 59 sera from family contacts of PTB, and $\mathbf{4 0}$ sera from cases of lung infections other than tuberculosis collected from September to December 2003 were used for the kit evaluation. METHODS AND MATERIALS: Enzyme-linked immuno sorbent assay test using tuberculosis A-60 antigen-based kit manufactured by Anda Biologicals, France was used for the evaluation. STATISTICAL ANALYSIS: Differences in the optical density (OD) values for immunoglobulins $G$ (IgG), and immunoglobulins $M$ (IgM) antibodies in various groups were studied using t-test. RESULTS: On the basis of the findings the threshold value was setup as $400 \mathrm{U}$ for IgG and mean $O D$ for sera from healthy volunteers $+2 S D$ as the threshold for IgM. The sensitivity was $80 \%$ and specificity $95.8 \%$ for the IgG antibody test. The efficiency and predictive values were also high. The sensitivity for IgM was low (28.5\%) but the specificity was high (95.7\%). None of the 40 nontubercular lung infection cases were positive for the $\lg G$ and $\lg M$ antibody test for A-60, whereas five and three cases of 59 family contacts of PTB were positive for IgG and IgM antibody test. The test reproducibility was good for both IgG and IgM. CONCLUSION: IgG antibody test using A-60 antigen has good sensitivity and specificity, whereas IgM antibody test had high specificity but low sensitivity. Multicentric trials suggested evaluation of the diagnostic utility of the test for the extra-PTB.

KEY WORDS: A-60 antigen; ELISA; IgG; IgM; tuberculosis

India with 14 million cases of tuberculosis carries one-third of the global load of the

\section{Department of Microbiology and Immunology,} Choithram Hospital and research Centre, Indore, India

\section{Correspondence}

Dr DS Chitnis

Department of Microbiology and Immunology,

Choithram Hospital and research Centre,

Manik Bagh Road, Indore - 452014
Maithram

India

E-mail: ds_chitnis@rediffmail.com demands high-level skill, expensive setup, and reagents. Further, all the above methods demand sample from site of infection and in many extra pulmonary situations may not be possible. Hence, serological diagnosis has attracted the attention of laboratories all over the world.

An array of commercial kits to detect antibodies against $\mathrm{A}-60,38 \mathrm{kDa}$ and other antigens such as ES-31 antigen, KP-90 antigen, Lipoarabinomannan, and many more antigens have been largely evaluated in the Western countries and the threshold value has been setup on the basis of the Western population, where endemicity is low and mass Bacillus Calmette Guerin (BCG) vaccination not practiced. Hence, local evaluation before marketing the kits in India is required.

The A-60 antigen-based kit was selected for evaluation, because it has been used widely in the country as evident with the Indian literature. ${ }^{[2]-[12]}$ The antigen A-60 complex is a major mycobacterial antigen and the main component of reference tuberculin RT23. ${ }^{[13]} \mathrm{A}$ 60 antigen is extracted from Mycobacterium bovis strain BCG. It is composed of proteins, carbohydrate, and lipid in roughly equal amount. ${ }^{[14],[15]}$ The purpose of the present study is to evaluate the A-60 antigen-based enzymelinked immuno sorbent assay (ELISA) kit using sera from normal local population, bacteriologically proved pulmonary tuberculosis (PTB) cases, nonsymptomatic family contacts of PTB cases, and nontubercular lung infection cases to evaluate sensitivity, specificity, efficiency, reproducibility, and predictive value. The serological diagnosis can be of value for extra-PTB. However, bacteriologically confirmed cases of extra-PTB are not easily available and hence, only bacteriologically confirmed cases of PTB were included in the present study for the test evaluation.

\section{METHODS}

The four groups of cases included in the present study were healthy volunteers, bacteriologically confirmed cases of PTB, healthy contacts of PTB cases, and cases of lung infections other than tuberculosis.

Healthy volunteers: healthy voluntary blood donors from Indore city and the adjoining area who came for blood donation from September to December 2003 at the Blood Bank of Choithram Hospital and Research center were included as normal population. All the 114 volunteers ( 89 men and 25 women) were in the age group of 21-50 years and neither had history of any notable infection in the past 2 years, nor had symptomatic tuberculosis in the lifetime. Tuberculin test, however was not carried out on them.

Bacteriologically confirmed cases of PTB

One hundred and five active cases of PTB who approached at Choithram hospital dispensary from September to December 2003 were included in the study. The inclusion criteria were: (i) active PTB infection, based on clinical, radiological and sputum Acid Fast Bacillus (AFB) smear positivity. All the cases had cough (>3-week duration) with expectoration, weight loss, fever, tiredness, 
loss of appetite, breathlessness, and chest pain was reported by the majority and haemoptosis noted in 21 cases. The smears of sputum samples revealed $>10-99 \mathrm{AFB} / 100$ oil immersion fields and scored as $1+$ or greater based on Auramine $O$ staining. The chest X-rays showed a classical pattern of upper lobe infiltrate/bilateral infiltrate/cavitation with or without pulmonary fibrosis and shrinkage. (ii) A new case who came to the clinic with no history of anti-TB drugs and in the age group of $16-50$ years. Four children below 16 years were not able to give sputum samples and hence excluded, whereas five cases above 50 years were excluded because the history revealed the infection as a recurrence or as a relapse.

The exclusion criteria were PTB cases on chemotherapy and new cases not willing for informed consent.

The symptomatic phase before coming to the clinic ranged from 1 month to 1 year. The blood samples for the serology were collected within 1 week of documentation of AFB in sputum and before starting chemotherapy. The group comprised of 70 men and 35 women. The PTB status was further confirmed by AFB culture on Lowenstein-Jensen medium after alkali concentration of sputum samples.

Family contacts of PTB cases:

Blood samples from 59 family contacts ( 30 men, 29 women) of PTB cases were also included as controls. The family contacts were spouses; apparently healthy with no symptoms, and blood samples were given after
6 months of the detection of index PTB case in the family.

Cases of lung infections other than tuberculosis:

Another control group of 40 cases (24 men, 16 women) had lung infection wherein tuberculosis was ruled out on the basis of AFB smear negativity in three consecutive samples and subsequently AFB culture negativity.

Tuberculin test was carried out on all subjects from PTB group, family contacts, and nontubercular lung diseases group using $5 U$ purified protein derivative (PPD) (Span diagnostics, India).

Sera samples from all the subjects were given code numbers and stored at $-700^{\circ} \mathrm{C}$ till the day of testing for the antibody test. Decoding was done at the time of data analysis.

Commercially available IgG and IgM antibody test manufactured by Anda Biologicals (67067 STRASBOURG Cedex, France) was used in the study. The test detects antibodies to A-60 antigen coated over the wells. The ELISA test was carried out as described in the manufacturer's protocol. The sera samples and the kit reagents were allowed to come to room temperature before the test. Positive and negative controls provided in the kit were run for every batch of ELISA test.

The sera samples were diluted $1: 100$ in the sample diluent. The diluted samples and controls added $(100 \mathrm{ml})$ to the wells and incubated at $370^{\circ} \mathrm{C}$ per $1 \mathrm{~h}$. The wells washed five times before adding anti human IgG- or IgM-peroxidase conjugate. After $30 \mathrm{~min}$ at $370^{\circ} \mathrm{C}$ the wells washed again five times and $100 \mathrm{ml}$ tetramethylbenzidine substrate was added. The reaction was stopped by the addition of $100 \mathrm{ml} \mathrm{H} 2 \mathrm{SO} 4$ after $15 \mathrm{~min}$ incubation at $370^{\circ} \mathrm{C}$ and absorbance measured at $450 \mathrm{~nm}$ using MRX-ELISA (Dynex, USA) plate reader.

The hospital ethical committee had approved the study and informed consents from patients and volunteers were taken before including them in the study.

\section{Statistical methods}

IgM cut-off sample and IgG $4 \mathrm{U} / \mathrm{ml}$ reference standard provided in the kit were run in duplicate consecutively (weekly) for 10 weeks to plot the variation with reference to 1 and 2SD. For making scatter diagram for sera from healthy volunteers and from PTB cases, optical density (OD) values for the sera were plotted to study dispersal of the range. Optical density values for sera from PTB cases, healthy volunteers, family contacts, and nontubercular lung cases were used to determine sensitivity, specificity, and efficiency using the standard formulae. ${ }^{[16]}$ Predictive values were calculated as a measure of value of a test in relation to the prevalence of disease in the population. ${ }^{[16]}$ Differences in OD values of sera from various groups were checked for statistical significance, using two tailed, two sample unequal variance $t$-test.

\section{RESULTS}

Mycobacteria were isolated from all the 105 sputum samples from PTB cases. Ninety-nine of the isolates were Mycobacterium tuberculosis complex (Niacin test positive), whereas six were mycobacteria other than tuberculosis (MOTT). None of the sputum samples from nontubercular lung infection cases revealed the growth of mycobacteria. The microbial spectrum of the nonmycobacterial agents from sputum/BAL samples in the group is depicted in Table 1.

Five of the cases had underlying diabetes, whereas 15 had chronic asthma. Infiltrative lung disease was noted in the radiological/ bronchoscopy examination. Sarcoidosis was not seen in any of the cases.

Tuberculin test was positive among 84/105 PTB cases, 12/59 contacts of PTB cases, and four of the nontuberculosis lung infection cases.

Mean OD values in ELISA test for A-60 antibodies for sera from PTB cases, healthy volunteers, family contacts of PTB cases, and nontubercular lung infection group are given in

Table 1: Spectrum of microbial agents in lung infections other than tuberculosis from sputum/BAL samples $(n=40)$

\begin{tabular}{ll}
\hline Organism & No. of isolates \\
\hline Candida albicans & 3 \\
Klebsiella pneumoniae & 11 \\
Staphylococcus aureus & 5 \\
Pseudomonas aeurgenosa & 6 \\
Haemophilus influenzae & 3 \\
Escherichia coli & 5 \\
Streptococcus pneumoniae & 5 \\
\hline Five of the cases had underlying diabetes, whereas 15 had \\
chronic asthma. Infiltrative lung disease was noted in the \\
radiological/bronchioscopy examination. Sarcoidosis was not
\end{tabular}
radiologica/bronchioscopy examination. Sarcoidosis was seen in any of the cases. 
Table 2: Mean OD for A-60 TB ELISA antibody test for bacteriologically confirmed PTB cases, healthy volunteers, PTB Family contacts cases, and nontubercular lung infection cases

\begin{tabular}{lllll}
\hline Groups & $\lg G$ & & IgM & \\
\hline & Mean & SD & Mean & SD \\
Healthy volunteers $(n=114)$ & 0.67 & 0.31 & 0.62 & 0.25 \\
Bacteriologically confirmed PTB cases $(n=105)$ & 1.30 & 0.39 & 0.86 & 0.29 \\
PTB family contacts cases $(n=55)$ & 0.89 & 0.19 & 0.73 & 0.06 \\
Non-tubercular lung infection cases $(n=40)$ & 0.63 & 0.06 & 0.62 & 0.07 \\
\hline
\end{tabular}

Table 2. Differences in mean OD among sera from healthy volunteers and PTB cases both for $\lg G$ and $\operatorname{lgM}$ were highly significant in two tailed, two sample unequal variance $t$-test $(P$ $<0.001$ and 0.001 , respectively). Similarly, differences among mean OD values for sera from healthy volunteers and family contacts of PTB cases were also statistically significant for $\lg G$ and $\lg M$ antibody ( $P$ values $<0.001$ and 0.002 , respectively). However, differences in mean OD values for healthy volunteers and the group of non-tubercular lung infection were not significant for IgG and IgM antibody to A-60 antigen $(P=0.44$ and 0.97 , respectively). The mean $+2 S D$ OD value for IgG and IgM among

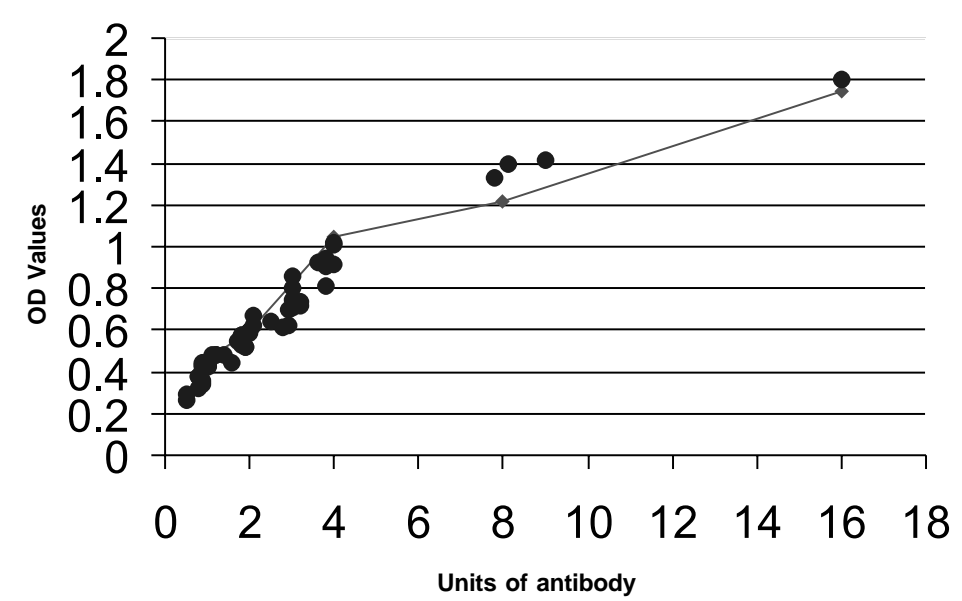

Figure 1: Dispersal of $\lg G$ antibody to A-60 among healthy volunteers. Series 1: Standard curve using $0,2,4,8$, and $16 \mathrm{U}$ standards. Series 2: Values of IgG among 114 sera from healthy volunteers. Number of points appears less due to superimposition sera from healthy volunteers was considered as threshold to report as positives.

Scatter diagram for $\lg G$ antibody to A-60 among healthy volunteers is displayed in Figure 1. Standard curve using OD values for $2,4,8$, and 16 units of standard IgG against OD values is shown in the background and it is evident that only four sera among healthy volunteers were outliers (value above $400 \mathrm{U}$ $\mathrm{ml}$ considering $1: 100$ dilution of the serum). Scatter diagram for $\lg G$ values among PTB cases is shown in Figure 2. Eighty-four samples had IgG levels above $4 \mathrm{U}$ (for $1: 100$ serum dilution) (values superimposed over ten

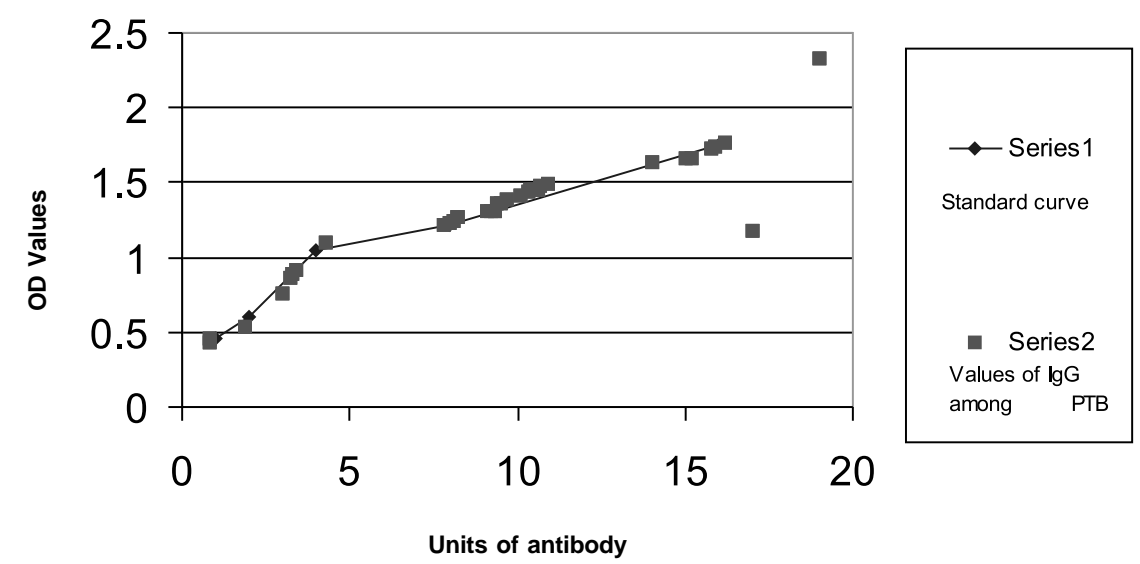

Figure 2: Dispersal of IgG antibody to A-60 among PTB cases. Series 1: Standard curve using 0,2, 4, 8, and $16 \mathrm{U}$ standards. Series 2: Values on sera from 105 PTB cases. Number of points appears less due to superimposition

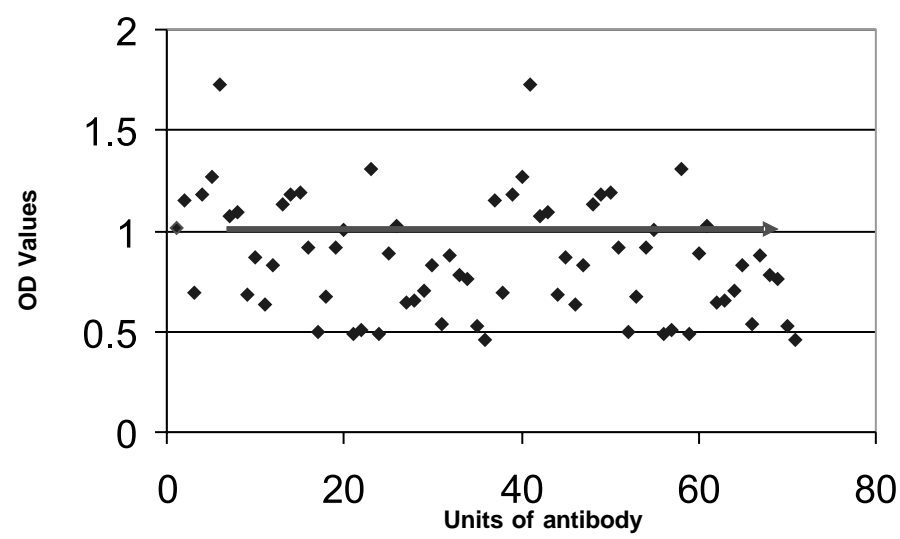

Figure 3: Dispersal of lgM antibody to A-60 among PTB cases. Horizontal line corresponds to OD values of the cutoff sample. Optical density values for 105 sera from PTB cases. Number of points appears less due to superimposition

points). In case of IgM, antibody levels were above the threshold for 30 sera [Figure 3]. The scatter diagram also supports the threshold levels setup on the basis of mean $+2 \mathrm{SD}$ values

for $\lg G$ and $\lg M$ antibody test.

Positivity, sensitivity, and specificity for $\lg G$ and $\lg M$ antibody: $\lg G$ and $\lg M$ positivity in various

Table 3: $\lg G$ and $\lg M$ antibody positivity among PTB and control groups

\begin{tabular}{lllll}
\hline A-60 antibody class & PTB $(\mathrm{n}=105)$ & Healthy volunteers $(\mathrm{n}=114)$ & Contacts of PTB $(\mathrm{n}=59)$ & $\begin{array}{l}\text { Cases of nontubercular } \\
\text { lung infections }(\mathrm{n}=40)\end{array}$ \\
\hline lgG positive & $84(80.0 \%)$ & $4(3.5 \%)$ & $5(8.4 \%)$ & 0 \\
lgM positive & $30(28.6 \%)$ & $6(5.2 \%)$ & $3(5.0 \%)$ & 0 \\
\hline
\end{tabular}


groups is shown in Table 3. Eighty-four $\lg G$ positives and $30 \mathrm{lgM}$ positives in PTB group were considered as true positive, whereas the positive samples among healthy volunteers and contact of PTB were considered as false positives. Twenty $\lg G$ negative and $75 \lg M$ negative samples in PTB group were considered as false negatives. The sensitivity for $\operatorname{lgG}$ antibody test was $80 \%$, specificity was $95.8 \%$, efficiency $90.5 \%$, and positive predictive value $90.3 \%$. In case of $\lg$, antibody sensitivity was as low as $28.6 \%$ but specificity was $95.7 \%$, efficiency was $73.6 \%$, and positive predictive value was $77 \%$.

Reproducibility check is essential as a quality control measure. The IgM reference cut-off was run weekly for ten consecutive weeks and the dispersion was studied. Almost all values lay within 1SD and only one value was closer to $2 S D$. In case of IgG, $4 \cup$ reference standard was run weekly for ten consecutive weeks and the results were within 1SD for all the tests, whereas only two points were proximal to 2SD. Therefore, good reproducibility was observed for IgG and IgM controls.

\section{DISCUSSION}

Comorbidity of HIV and tuberculosis is well known. In the present series, the PTB cases included were part of the anonymous HIV surveillance program and three of the 105 subjects were HIV antibody reactive (not mentioned in results).

Tuberculin test is an indicator of cell-mediated immune response to tuberculosis. Eighty-four of the bacteriologically confirmed cases were positive in tuberculin test and the same number of the sera samples were positive for IgG antibody. However, 15 of them showed discrepancy, that is, antibody test positive but tuberculin test negative and vice versa. It also needs to be pointed out that 12 subjects among PTB contacts and four cases of nontubercular lung disease cases were tuberculin test positive but negative for $\lg G$ and IgM antibody to A-60.

The contacts of PTB cases included in the study were spouses of the index PTB cases and five of them were IgG positive, whereas three were IgM positive. The positivity could be due to exposure resulting in nonsymptomatic infection or the immune response without getting active infection. Further follow up in relation to development of active infection was not done.

It would have been interesting to study lgG and IgM levels to A-60 antigen among treated PTB cases. However, only 24 treated cases were available for follow up (results not included in 'result' part). None of them had IgM antibody to A-60 above cut-off, whereas IgG antibody was positive for all the cases. In six of the cases, IgG level was reduced compared to the initial value before anti-tubercular treatment while IgG level was found to increase in 18 cases. Reduction in IgG level could be due to the waning of the antigen following effective therapy and increased IgG level correlated with the continued immune response subsequent to the initial lgG level. Sero-diagnosis of tuberculosis using various antigens-based ELISA tests has been reviewed by Bhatia et al.[10] The review mentioned A-60-based IgG antibody test to be more sensitive and specific than IgM antibody. The $38 \mathrm{kDa}$ antigen-based tests were reported to be specific but less specific. Uma et al. ${ }^{[17]}$ reported sensitivity as $61 \%$ for IgG and only $10 \%$ for IgM antibody using $38 \mathrm{kDa}$ antigen and Mathai et al. ${ }^{[18]}$ compared five different antigens-based commercial kits and the sensitivity varied from 46 to $68 \%$. Meena et al. ${ }^{[3]}$ reported A-60- and LPS-based tests to be more sensitive and specific than $38 \mathrm{kDa}$ and KP-90-based ELISA tests.

It appears that the Indian experiences are more with A-60 antigen-based kits. ${ }^{[2]-[12]}$

The threshold values used by Munshi et al. ${ }^{[9]}$ was $200 \mathrm{U}$ and Maheshwari et al. ${ }^{[6]}$ had selected $250 \mathrm{U}$ as threshold. In the present study, threshold had to be raised to $400 \mathrm{U}$. Therefore, setting up of threshold values for the individual laboratory based on the local population is required for adequate sensitivity and specificity.

The duration of illness and the age group also need to be considered for the negative antibody tests. Ahmed et al. ${ }^{[2]}$ pointed out lower positivity for age group 1-14 years and duration of less than 3 months in case of tuberculous lymphadenitis. The class of the antibody is also an important factor. Immunoglobulin $\mathrm{G}$ holds the great promise in diagnosis of active tuberculosis both in children and in adults. ${ }^{[10]}$ Sensitivity and specificity for $\lg$ antibody to A-60 was found to be in the range of $75-100 \%$ in various other Indian reports. ${ }^{[2]-[12]}$ However, proper evaluation, setting up of thresholds, and reproducibility study was lacking in most of the studies.
The IgM antibody is the first to appear for any antigen and therefore could be expected to be of diagnostic value for the recent tuberculosis. However, in case of $\operatorname{lgM}$ antibodies test, the sensitivity was low $(28.5 \%)$ in the present study. This possibly could be due to inclusion of PTB cases with a symptomatic history of 112 months. As a result of longer duration of infection, the $\operatorname{lgM}$ antibodies might have declined in the proportion of PTB cases. Longer incubation period in case of tuberculosis may result in decline of IgM before symptoms appear. However, the IgM antibody appears to be less sensitive as evident in the present study and also has been reported by others. ${ }^{[6],[10],[12]}$ Bhatia et al. ${ }^{[10]}$ reported IgG sensitivity of $94 \%$ and IgM sensitivity of only $33 \%$ in extra-PTB, whereas Maheshwari et al. ${ }^{[6]}$ observed IgG sensitivity of $75 \%$ and $\operatorname{lgM}$ sensitivity of $37.5 \%$ in tuberculoma cases. A limitation of the present study has been noninclusion of extra-PTB cases. Hence, the multicentric trials on the diagnostic utility of the test on cases of extra-PTB are suggested.

To conclude, the threshold value setup for IgG antibody to A-60 antigen was $400 \mathrm{U} / \mathrm{ml}$. The $\lg$ antibody test had sensitivity of $80 \%$ and specificity of $95.8 \%$. The sensitivity for $\lg M$ antibody was low (28.6\%), although the specificity was high (95.7\%). The ELISA kit also showed good reproducibility.

\section{ACKNOWLEDGMENTS}

We are thankful to the management of Choithram Hospital and Research Center Indore (MP) for providing the facility for the research work. Special thanks to Dr. Santosh Chobe and Dr. B. S. Chadda from Choithram 
Dispensary, Indore for providing the blood samples of PTB cases.

\section{REFERENCES}

1. Miormer H. Diagnosis of pulmonary tuberculosis. Lancet 1994;344:127.

2. Ahmed Z, Pandey DK and Beg M. Role of AntiA60 IgG titers were measured in 120 FNAC confirmed patients of tuberculous lymphadenitis. Indian J Tuber 2002;49:101.

3. Meena LS, Goel S, Sharma SK, Jain NK, Banavaliker JN, Bedwal RS, Singh Y. Comparative study of three different polysaccharide antigens for the serodiagnosis of tuberculosis. J Clin Lab Anal 2002;16:151-5.

4. Anuradha S, Kaur R, Singh NP, Baveja UK. Serodiagnosis of extra pulmonary tuberculosis using A-60 antigen. J Commun Dis 2001;33:126.

5. Ghoshal U, Kishore J, Kumar B, Ayyagari A. Serodiagnosis of smear and culture negative neuro-tuberculosis with enzyme linked immunosorbent assay for anti A-60 immunoglobulins. Indian J Pathol Microbiol 2003;46:530-4.

6. Maheshwari A, Gupta HL, Gupta S, Bhatia R, Datta KK. Diagnostic utility of estimation of mycobacterial antigen A60 specific immunoglobulins in serum and CSF in adult neurotuberculosis. J Commun Dis 2000;32:5460.

7. Mahajan M, Singh NP, Gadre DJ, Talwar V, Gupta HC, Agarwal DS. Detection of IgM antibodies in pulmonary tuberculosis by ELISA using A60 antigen. J Commun Dis 1996;28:17680.

8. Gupta A, Kumar V, Xess A, Sharma HP, Shahi SK. Role of enzyme linked Immunosorbent assay in the diagnosis of suspected cases of genitourinary tuberculosis. Indian J Pathol Microbiol 1999;42:307-9.
9. Munshi MM, Chiddarwar S, Patel A, Grover S. Serodiagnosis of Extra-pulmonary tuberculosis by ELISA. Indian J Pathol Microbiol 1993;36:35660.

10. Bhatia AS, Kumar S, Harinath BC. ImmuneDiagnosis of Tuberculosis: An update. Indian $\mathrm{J}$ Clin Biochem 2003;18:1-5.

11. Singh $P$, Bhaveja $C P$, Talukdar $B$, Kumar $S$, Mathur MD. Diagnostic utility of ELISA test using antigen A60 in suspected cases of tuberculosis meningitis in Pediatric age group. Indian J Pathol Microbiol 1999;42:11-14.

12. Gupta S, Kumari S, Banwalikar JN, Gupta SK. Diagnostic utility of the estimation of mycobacterial Antigen A60 specific immunoglobulins $\lg M, \lg A$ and $\lg G$ in the sera of cases of adult human tuberculosis. Tuber Lung Dis $1995 ; 76: 418-24$

13. Harboe M. Antigen of PPD, old tuberculin and autoclaved mycobacterium bovis BCG studied by Immunoelectrophoresis. Am Rev Respir Dis 1981;124:80-7.

14. Fabre I, L'Homme O, Bruneteau M, Michel G and Cocito $\mathrm{C}$. Chemical composition of antigen 60 from M. bovis BCG. Scand J Immunol 1986;24:591-602.

15. Cocito C, Vanlinden F. Preparation and properties of antigen 60 from mycobacterium bovis. Clin Exp Immunol 1986;66:262-272.

16. Baveja UK, Chattopadhaya D, Aggarwal RK. Evaluation of HIV kits and preparation of serum panel. In: Baveja UK, editor. HIV testing manual: Laboratory diagnosis, bio-safety and quality Control. National Institute of Communicable Diseases. NACO; 2002. p. 107-113.

17. Uma Devi K R, Ramalingam B, Brennan PJ, Narayanan PR, Raja A. Specific and early detection of $\lg G, \lg A$ and $\lg M$ antibodies to Mycobacterium tuberculosis $38 \mathrm{kDa}$ antigen in pulmonary tuberculosis. Tuberculosis 2001;81:249-53.

18. Mathai E, Rajkumari R, Kuruvilla $P$ J, Henry $K$
Brahmadathan KN, Inbamalar U, Abraham OC.

Evaluation of serological test for the diagnosis

of tuberculosis. Indian J Pathol Microbio 2002;45:303-5.
Indian Journal of Medical Sciences is pleased to announce the launch of its website. The URL of the website is http://www.indianjmedsci.org.

The features of the site are:

- Free full text availability of articles in HTML as well as PDF

- Link to abstracts and full text from the cited references

- Link to PubMed abstracts of published articles by authors

- Link to related articles in PubMed

- Link from text of articles to various databases and search engines

- Facility to submit comments on articles

- Email notifications on new issue release

- Statistics of articles download and visits

- Structure based on OpenURL, DC Metadata and other international standards 